\title{
New measures and concepts in the control and prevention of COVID-19:What we need to know
}

\author{
Gaohong $\mathrm{Wu}^{1}$, Ping Jiang ${ }^{2}$, Huan $\mathrm{Yuan}^{3}$, Xueping Zhu ${ }^{3}$, and Yuan $\mathrm{Shi}^{4}$ \\ ${ }^{1}$ Children's Hospital of Soochow University \\ ${ }^{2}$ Bayingolin Mongolian autonomous prefecture people's hospital, Korla, Sinkiang 841000, \\ China \\ ${ }^{3}$ Children's Hospital of Soochow University, China \\ ${ }^{4}$ Children's Hospital, Chongqing Medical University, Ministry of Education Key \\ Laboratory of Child Development and Disorders, Key Laboratory of Pediatrics in \\ Chongqing, Chongqing, China Chongqing 400014, China
}

April 28, 2020

\begin{abstract}
The emergence and outbreak of coronavirus disease 2019 (COVID-19) poses great challenges to our society, economy, and public health, and has already become an international public health emergency. The prevention and control of COVID-19 requires early detection and the timely and effective control of virus dissemination. Front-line medical personnel in the outpatient and emergency departments of hospitals, quarantine centers, and entry and exit ports are key to the early detection and control of COVID-19. Based on experiences in the prevention and control of other new and recurrent infectious diseases, epidemiologists previously proposed the concept of "vital sign zero" and the "Identify-Isolate-Inform" (3I) system for the detection and control of infectious diseases. The use of "vital sign zero" and the "3I system" for COVID-19 will facilitate the prevention and control of new infections, provide more timely treatment and effective isolation of patients, and protect the safety and health of medical workers. These concepts will also prevent the spread of COVID-19 and help to ensure public health and safety.
\end{abstract}

New measures and concepts in the control and prevention of COVID-19 :What we need to know

Running Head: A review on the prevention of COVID-19

Gaohong $\mathrm{Wu}, \mathrm{MM}^{1 *}$, Ping Jiang, $\mathrm{MB}^{1,2}$, Huan Yuan, $\mathrm{MB}^{1}$, Xueping Zhu, MD ${ }^{1}$, Yuan Shi, MD, PhD ${ }^{1.3}$

1 Department of Neonatology, Children's Hospital of Soochow University, China

2 Department of pediatrics, Bayingolin Mongolian autonomous prefecture people's hospital, Korla, Sinkiang 841000, China

3 Department of Neonatology, Children's Hospital, Chongqing Medical University, Ministry of Education Key Laboratory of Child Development and Disorders, Key Laboratory of Pediatrics in Chongqing, Chongqing, China Chongqing 400014, China

${ }^{1}$ These authors contributed equally to this work and wish to be considered as co-first authors

*Corresponding author:

Gaohong Wu, M. M., Department of Neonatology, Children's Hospital of Soochow University 
NO.92 Zhongnan Street, Industrial Park, Suzhou 215025, Jiangsu, China

Tel: $+86-13041850698$

Email: 1217555215@qq.com

Abstract: The emergence and outbreak of coronavirus disease 2019 (COVID-19) poses great challenges to our society, economy, and public health, and has already become an international public health emergency. The prevention and control of COVID-19 requires early detection and the timely and effective control of virus dissemination. Front-line medical personnel in the outpatient and emergency departments of hospitals, quarantine centers, and entry and exit ports are key to the early detection and control of COVID-19. Based on experiences in the prevention and control of other new and recurrent infectious diseases, epidemiologists previously proposed the concept of "vital sign zero" and the "Identify-Isolate-Inform" (3I) system for the detection and control of infectious diseases. The use of "vital sign zero" and the "3I system" for COVID19 will facilitate the prevention and control of new infections, provide more timely treatment and effective isolation of patients, and protect the safety and health of medical workers. These concepts will also prevent the spread of COVID-19 and help to ensure public health and safety.

Key words: Severe acute respiratory syndrome coronavirus 2; Coronavirus disease 2019; Control and prevention; vital sign zero; identify-isolate-inform system

The outbreak of severe acute respiratory syndrome coronavirus 2 (SARS-CoV-2), which originated in Wuhan, China, has become a major global health issue. This novel coronavirus can cause severe respiratory tract infections and lead to bronchiolitis or pneumonia, a disease named coronavirus disease 2019 (COVID-19) by the World Health Organization (WHO) on Feb 12, 2020. Because of the high prevalence of SARS-Cov-2 infections, the WHO declared this an international public health emergency on Jan 30, 2020 (Huang et al., 2020). This virus is highly contagious and its basic reproduction number $\left(\mathrm{R}_{0}\right)$ is 3.58 (Chen et al., 2020). By Mar 16, 2020, this virus infected more than 81,000 people world-wide and caused 3200 deaths, corresponding to a mortality rate of approximately $4.0 \%$. So far, there are no specific treatments for COVID-19, but many public health measures have been implemented to improve control and prevention. Because health care workers are facing the potential need to assess many patients with COVID-19 new measures and concepts — "vital sign zero" and the "Identify-Isolate-Inform" (3I) system — will help to assure the timely treatment and isolation of patients, guarantee the safety and health of medical staff, more closely monitor and prevent the spread of this infectious disease, and ensure public health and safety.

\section{Vital sign zero}

Use of triage to prevent and control COVID-19

Triage refers to judging the severity of a disease according to patients' main symptoms and signs, and arranging treatments accordingly. An important basis of triage is the recording of vital signs, including pulse, blood pressure, respiration, and heart rate. In addition, elevated body temperature and respiratory symptoms are common in COVID-19, and are closely related to disease severity. Therefore, health care workers consider body temperature and respiratory symptoms when implementing triage for patients with COVID-19. When a new infectious disease emerges, health care workers use triage for the early detection of infected patients, prevention of disease spread, and protection of the safety of medical staff. In an outpatient department, and especially in an emergency department, triage is an important procedure for managing multiple patients with infectious diseases. The outbreak and spread of the Middle East respiratory syndrome (MERS) in South Korea during 2015 was due to the limited understanding of this new disease, the lack of effective prevention and control measures, and the failure to rapidly identify and isolate patients. Missed diagnoses may also lead to infection and spread of a disease among medical staff. Therefore, Koenig proposed that when health care workers encounter a patient who may have an infectious disease, certain information should be collected during triage and before recording the patient's vital signs, a concept she called "vital sign zero" (Koenig, 2015). Therefore, to best control the COVID-19 epidemic, medical staff must first have the answers to certain "vital sign zero" questions when encountering a new patient. In 
particular, medical staff should inquire about the medical history, identify potentially exposed patients as early as possible, and implement measures of isolation and protection. Thus, medical staff should integrate the concept of "vital sign zero" into the COVID-19 triage system, maintain a high degree of vigilance to COVID-19, try to identify COVID-19 patients as soon as possible, and implement measures that reduce the risk of disease transmission.

Vital sign zero

"Vital sign zero" is a new concept that can help prevent and control infectious diseases. It refers to the collection of certain relevant information about a patient by medical staff prior to the recording of the patient's vital signs. Specifically, medical workers should first assess the risk of infectious disease, determine whether the patient may suffer from an infectious disease, and assess the potential harm of the infectious disease. They should then take appropriate personal protective measures to prevent their own infection. Patients with infectious diseases should be isolated in a timely manner according to the required prevention and control measures (Kristi et al., 2016).

The application of "vital sign zero" can help medical staff prevent and control the dissemination of infectious diseases. In particular it can help them to identify patients with the greatest potential for having an infectious disease, to use personal protective equipment in an appropriate and timely manner, and to protect their own health and safety, one of the most significant considerations when encountering a new infectious disease or a possibly infectious disease (Koenig, 2015). The procedures recommended by the "vital sign zero" concept should become routine for all medical worker. This concept emphasizes that when medical workers treat patients, they must pay attention to their own safety and health, and should not blindly try to rescue patients while ignoring their own safety.

The ethics of vital sign zero

Will increasing the evaluation of patients who may suffer from infectious diseases during triage affect the timely treatment of these patients? How can health care workers balance the benefits of providing rapid treatment of patients, protecting the health of medical staff, and preventing the spread of infectious diseases? These are all new questions that must be addressed when caring for patients with COVID-19.

Medical workers around the world have increasing daily working hours and workloads, and this has led to occupational health problems. These include physical injuries, such as musculoskeletal disorders, and infectious diseases such as Acquired Immune Deficiency Syndrome (AIDS) and tuberculosis, and especially new infectious diseases, all of which threaten the health of medical staff and increase occupational stress (BeyMarrié, Engel, \& Abdullahi, 2018)

Looking back on the SARS epidemic in China during 2003, there was a lack of vigilance and failure to use necessary personal protection measures by medical staff. Thus, about 1000 medical workers developed SARS, and these workers accounted for about 1 of 5 cases and 1 of 3 of deaths. In February 2014, an outbreak of Ebola occurred in Africa. As of August 2014, more than 240 medical and nursing personnel were infected, and more than 120 people died (Le, \& Sorvillo, 2018)

Since late December 2019 when the first SARS-Cov-2 infection was reported in China, this infection has spread many other countries, and the epidemic has become a pandemic. As of February 11, the China Health Commission reported that 1716 cases were confirmed among medical workers, accounting for $3.8 \%$ of all confirmed cases. Six of these individuals died, accounting for $0.4 \%$ of all deaths. Among the 1716 cases, 1502 were medical workers in Hubei and 1102 cases were in Wuhan. Thus, it is very important to ensure the safety and health of medical staff, and at the same time save lives and support patients. The WHO examined the issues affecting medical staff, and pointed out that the lack of personal protective measures, the irregular use of medical equipment, insufficient staff size, and the long working hours in isolated areas has led to the infection of a large number of medical personnel (Hong-Xia et al., 2019). It is the mission of medical staff to prevent suffering and to save the sick and wounded; however, while actively treating patients, they must also balance the need for their own safety. Medical staff cannot blindly treat patients and ignore their own 
safety. It is a huge loss to patients and other medical workers when medical staff become infected and can no longer treat patients. Therefore, the concept of "vital sign zero" should be applied in the treatment of COVID-19. Combining "vital sign zero" with relevant policies, and establishment of early warning and timely notification systems will greatly reduce the threat of COVID-19 to public health.

\section{Overview of the Identify-Isolate-Inform system}

It is also necessary to establish a rapid, simple, and effective system for the identification and management of infectious diseases in the emergency rooms and outpatient departments of hospitals and to monitor individuals who may import or export the virus to different places. Medical staff must identify patients during the early stages of disease, and promptly implement prevention and control procedures to prevent spread of the infection. The "3I system" is a set of early detection and prevention methods that was designed to prevent and control the Ebola outbreak. It was designed by Koening and approved by the Ebola expert group of the American Association of Emergency Physicians (Koenig, 2015). It was finally distributed to emergency rooms nationwide by the U.S. Centers for Disease Control and Prevention on Nov 5, 2014. The "3I system" was subsequently modified and successfully used for the prevention and control of MERS, measles, Zika, mumps, hepatitis A, and other infectious diseases. The COVID-19 pandemic has been a public health disaster that has affected people all over the world. It is very important to prevent COVID-19 before an effective treatment plan is developed. Based on the previous "3I system", most regions in China have formulated a "3I system" for COVID-19 prevention and control (Table 1). The results indicated this program was successful, but this system needs further development and improvement.

\section{Identify}

The first step in the "3I system" is identification, i.e. identification of possibly infected persons (based on possible exposure or exposure history) and infected persons with any epidemiological risk factor. These individuals can be identified by on-site inquiry and examination. Medical personnel must record information on exposure history and contact history for the early detection of infected patients and possibly infected patients, and must be aware of this information to prevent missed or incorrect diagnoses.

One of the characteristics of the "3I system" is to display posters or even small cards that all medical staff can quickly read and understand. These displays provide basic information on the diagnosis and differential diagnosis of new infectious diseases. In this specific case, these displays can describe the methods used for detection and diagnosis of COVID-19, and make it easier for medical staff to identify potential patients. Early identification is the key to reduce the spread of COVID-19, so it is particularly important to apply these methods to prevent and control COVID-19 (Lai et al., 2020).

Isolate

Persons suspected of having an infectious disease, must be isolated locally where they are found, and contact with uninfected individuals must be prevented. Isolation involves the transfer of patients to other designated treatment sites or directly into isolation.

Health care workers must consider whether necessary isolation precautions should be implemented when evaluating patients. At the same time, they must decide whether isolation, quarantine, or surveillance is most appropriate. Isolation refers to the separation of symptomatic infected persons (patients) from uninfected persons to prevent the spread of disease. Quarantine refers to the separation of individuals who are not infected, but who were or may have been exposed to an infectious disease or pathogen, from individuals who were not exposed or are more susceptible to infection (Brooks et al., 2020). Surveillance is a population-based measure used to observe people who are likely to have been exposed to a disease, but have no symptoms. Surveillance, which is similar to quarantine but may allow the individual to be isolated at home, was used during the assessment of MERS infections (Koenig, Shastry, \& Burns, 2017). When observing patients during quarantine, the individual should be transferred into isolation when symptoms occur and infection is confirmed.

Diseases for which quarantine is suitable must pose a serious threat to public health, such as cholera, 
diphtheria, tuberculosis, plague, smallpox, yellow fever, viral hemorrhagic fever, and SARS (Koenig, 2012). Quarantine stations located at entry ports and national borders provide isolation as part of the national quarantine system to limit the introduction and spread of infectious diseases in a country. The duration of the quarantine is determined by the incubation period of the infection. At present, SARS-Cov-2 infected patients with symptoms of COVID-19 are the main sources of infection, but asymptomatic infected patients (no symptoms of COVID-19) may also be infectious (Xu et al.,). Therefore, the combination of quarantine and isolation is effective for the prevention and control of COVID-19, and this method has achieved good results in China (Figure 1).

Inform

"Inform" - timely reporting — is the third step of the "3I system". Thus, health care workers should promptly report the location where a patient is found and diagnosed to the appropriate hospital department, supervisor, and law enforcement officials. Clear reporting schemes must be developed and alternatives must be available in the "3I system". Thus, when suspected cases of COVID-19 are identified in China, direct online reporting is required within 2 hours, specimens are collected for PCR analysis, and the suspected individuals are immediately transferred to designated hospitals using safe transportation. These procedures have greatly reduced the number of missed cases.

\section{Application and significance of vital sign zero and the 3I system}

The combined use of "vital sign zero" and the "3I system" can ensure the safety of medical staff, provide early identification of infected patients, and prevent the spread of infectious diseases. When medical resources are limited, the medical staff should work to maximize the health and safety of the entire population, not only patients with known infecrtions (Macias, \& Hardy, 2019). Therefore, when encountering a new patient, health care workers should initially assess the likelihood of disease transmission ("vital sign zero") in an effort to protect health care workers and safety of the public. The use of "vital sign zero" at the time of triage allows early identification of high-risk groups and allows health care workers to use appropriate personal protective equipment. This is an important intervention that helps to manage existing emerging infectious diseases, such as COVID-19, and major infectious diseases that may arise in the future (Koenig, Alassaf, \& Burns, 2015) .

It is evident from the outbreak of COVID-19 that a rapid and effective response is the key for controlling outbreaks of infectious diseases (Wang et al., 2020). The WHO provides comprehensive definitions and treatment criteria for various infectious diseases, but these are not applicable to the first-line medical staff who perform rapid risk assessment. The "3I system" considers the epidemiological characteristics of an infectious disease and its main symptoms, and provides simple instructions for management of new infectious diseases by first-line medical staff and on-site epidemic prevention personnel. It is a simple and readily applicable method that can be used to rapidly detect and isolate infected patients and to block the spread of infectious diseases.

\section{Conclusion}

The early detection and prevention of COVID-19 should focus on hospital outpatients, emergency room patients, and individuals entering or leaving an area with known or suspected infections. The introduction and implementation of the concept of "vital sign zero" should be used before taking baseline measurements of breathing, heartbeat, blood pressure, and other vital signs. Based on the information collected, medical workers take appropriate personal protective measures that reduce the probability of becoming infected. At the same time, the "3I System" should be used to detect patients with COVID-19 as early as possible, so that isolation and reporting can be performed according to the predetermined plan to prevent the spread and reduce the prevalence of this disease. For potentially infectious patients, especially highly infectious patients, the "3I System" provides a simple and effective template for management. Because of COVID-19 and other emerging infectious diseases, the establishment of such a system could provide significant benefits to public health. 


\section{Acknowledgements}

Not applicable.

Ethics approval and consent to participate

Not applicable.

\section{Footnotes}

\section{Competing interests}

\section{The authors declare that they have no competing interests.}

\section{Authors' contributions}

GW participated in the study design and writing of the manuscript; PJ and HY participated in the English grammar correction and writing of the manuscript; XZ and YS participated in analysis and writing of the manuscript. All authors read and approved the final manuscript.

\section{Funding}

This study was financially supported by the The National Natural Science Foundation of China (NSFC, No. 81971423); The Jiangsu Provincial Maternal and Child Health Key Talents Project (No. FRC201731); Clinical Study of Prevention and Control System of New Coronavirus Infection in Neonates (SYS2020030).

Availability of data and materials

All data are fully available without restriction.

\section{References}

BeyMarrie, Schmidt., Engel, M. E., \& Abdullahi, L. (2018). Effectiveness of control measures to prevent occupational tuberculosis infection in health care workers a systematic review. Bmc Public Health, 7, 695695.

Brooks, S. K., Webster, R. K., Smith, L. E., Woodland, L., Wessely, S., Greenberg, N., \& Rubin, G. J. (2020). The psychological impact of quarantine and how to reduce it: rapid review of the evidence. Lancet, $395,912-920$.

Chen, T. M., Rui, J., Wang, Q. P., Zhao, Z. Y., Cui, J. A., \& Yin, L. (2020). A mathematical model for simulating the phase-based transmissibility of a novel coronavirus. Infect Dis Poverty, 9, 24.

Huang, C., Wang, Y., Li, X., Ren, L., Zhao, J., Hu, Y., Zhang, L., Fan, G., Xu, J., Gu, X., Cheng, Z., Yu, T., Xia, J., Wei, Y., Wu, W., Xie, X., Yin, W., Liu, M., Xiao, Y., Gao, H., Guo, L., Xie, J., Wang, G., Jiang, R., Gao, Z., Jin, Q., Wang, J., \& Cao, B. (2020). Clinical features of patients infected with 2019 novel coronavirus in Wuhan, China. Lancet, 395, 497-506.

Hong-Xia, L., Hui-Lan, Z., Jian-Ping, Y., \& Sha, Y. (2019). A Study on the Process of Donning and Doffing Personal Protective Equipment of Health Care Workers (HCWs) in China. Prehospital and disaster medicine: the official journal of the National Association of EMS Physicians and the World Association for Emergency and Disaster Medicine in association with the Acute Care Foundation, 34, s169-s169.

Koenig, K. L. (2015). Ebola Triage Screening and Public Health: The New "Vital Sign Zero". Disaster Med and Public Health Prep, 9, 57-58.

Kristi, K. L., Siri, S., Bandr, M., Almadhyan, A., \& Burns, M. J. (2016). Mumps Virus: Modification of the Identify-Isolate-Inform Tool for Frontline Healthcare Providers. West J Emerg Med, 17, 490-496.

Koenig, K. L. (2015). Health Care Worker Quarantine for Ebola: To Eradicate the Virus?or 
Alleviate Fear?. Ann of Emerg Med, 65, 330-331.

Koenig, K. L., Shastry, S., \& Burns, M. J. (2017). Hepatitis A Virus: Essential Knowledge and a Novel Identify-Isolate-Inform Tool for Frontline Healthcare Providers. West J Emerg Med, 18, 1000-1007.

Koenig, K. L. (2012). Crisis standard of care is altered care, not an altered standard. Ann Emerg Med, 59, $237-238$.

Koenig, K. L., Alassaf, W., \& Burns, M. J. (2015). Identify-isolate-inform: a tool for initial detection and management of measles patients in the emergency department. West J Emerg Med, 16, 212-219.

Le, Duc, J. W., Sorvillo, T. E. (2018). A Quarter Century of Emerging Infectious Diseases - Where Have We Been and Where Are We Going?. Acta Med Acad, 47, 117-130.

Lai, C. C., Shih, T.P., Ko, W. C, Tang, H. J., \& Hsueh, P. R. (2020). Severe acute respiratory syndrome coronavirus 2 (SARS-CoV-2) and coronavirus disease-2019 (COVID-19): The epidemic and the challenges. Int J Antimicrob Agents, 55, 105924.

Macias, G. R., \& Hardy, W. D. (2019). Spectrum of Diversity in Today's Infectious Diseases Workforce: It's Much Broader and Brighter Than You Think. J Infect Dis, 220, S42-S49.

Wang, L., Shi, Y., Xiao, T., Fu, J., Feng, X., Mu, D., Feng, Q., Hei, M., Hu, X., Li, Z., Lu, J., Tang, Z., Wang, Y., Wang, C., Xia, S., Xu, J., Yang, Y., Yang, J., Zeng, M., Zheng, J., Zhou W., Zhou, X., Zhou, X., Du, L., Lee, S. K., \& Zhou, W. (2020). Chinese expert consensus on the perinatal and neonatal management for the prevention and control of the 2019 novel coronavirus infection (First edition). Ann Transl Med, 8:47.

Xu, X. W., Wu, X. X., Jiang, X. G., Xu, K. J., Ying, L. J., Ma, C. L., Li, S. B., Wang, H. Y, Zhang, S., Gao, H. N., Sheng, J. F., Cai, H. L., Qiu, Y. Q., \& Li, L. J. (2020). Clinical findings in a group of patients infected with the 2019 novel coronavirus (SARS-Cov-2) outside of Wuhan, China: retrospective case series. BMJ, 368:m606.

Table 1. Application of the Identify-Isolate-Inform system for COVID-19.

\begin{tabular}{|c|c|c|}
\hline $\begin{array}{l}\text { Identify-Isolate-Inform (" } 3 \mathbf{I} \\
\text { system") Tools used by the }\end{array}$ & $\begin{array}{l}\text { Identify-Isolate-Inform (" } 3 \mathbf{I} \\
\text { system") Tools used by the }\end{array}$ & $\begin{array}{l}\text { Identify-Isolate-Inform ("3I } \\
\text { system") Tools used by the }\end{array}$ \\
\hline Emergency Department or & Emergency Department or & Emergency Department or \\
\hline Acute Respiratory Assessment & Acute Respiratory Assessment & Acute Respiratory Assessment \\
\hline $\begin{array}{l}\text { Service for assessment and } \\
\text { management of cases (patients } \\
\text { under investigation). }\end{array}$ & $\begin{array}{l}\text { Service for assessment and } \\
\text { management of cases (patients } \\
\text { under investigation). }\end{array}$ & $\begin{array}{l}\text { Service for assessment and } \\
\text { management of cases (patients } \\
\text { under investigation). }\end{array}$ \\
\hline Identify & Identify & Identify \\
\hline $\begin{array}{l}\text { Exposure history (within } 14 \\
\text { days) }\end{array}$ & Symptoms & Symptoms \\
\hline $\begin{array}{l}\text { Travel or residence in Wuhan and } \\
\text { surrounding areas or other } \\
\text { communities with case reports }\end{array}$ & $\begin{array}{l}\text { Suspected case: Any one } \\
\text { epidemiological factor and any } \\
\text { two clinical manifestations } \\
\text { without epidemiological history }\end{array}$ & $\begin{array}{l}\text {-Fever and/or respiratory } \\
\text { symptoms -Imaging features } \\
\text { consistent with COVID-19 } \\
\text {-During early-stage disease: } \\
\text { decreased/normal leukocyte and } \\
\text { lymphocyte counts }\end{array}$ \\
\hline
\end{tabular}

Contact with SARS-Cov-2

infected persons 
Exposure to patients with fever or respiratory symptoms from Wuhan and surrounding areas, or from communities with case reports.

Aggregative disease*

\section{Isolate}

-Put a surgical mask on the patient -Transfer the patient to a designated isolation ward -Use a face shield, eye protection, and other protection standards to prevent contact and air transmission -Use a tested N95 mask or device when entering a patient's room

\section{Inform}

Report to the following organizations immediately: -Hospital: take measures to prevent infection in time-Local health department
Confirmed case: Suspected case with pathogenic or serological evidence of infection

\section{Isolate}

-Put a surgical mask on the patient -Transfer the patient to a designated isolation ward -Use a face shield, eye protection, and other protection standards to prevent contact and air transmission -Use a tested N95 mask or device when entering a patient's room

\section{Inform}

Report to the following organizations immediately: -Hospital: take measures to prevent infection in time-Local health department
-Detection of SARS-Cov-2 by PCR -Gene sequencing suggests SARS-Cov-2 or highly homologous virus -SARS-Cov-2 specific $\lg \mathrm{M}$ antibody and $\lg \mathrm{G}$ antibody, or the SARS-Cov-2 specific IgG antibody changed from negative to positive or was 4-times or more higher than during the recovery period than the acute period

\section{Isolate}

-Put a surgical mask on the patient -Transfer the patient to a designated isolation ward -Use a face shield, eye protection, and other protection standards to prevent contact and air transmission -Use a tested N95 mask or device when entering a patient's room

\section{Inform}

Report to the following organizations immediately: -Hospital: take measures to prevent infection in time -Local health department

*Aggregative disease: 2 or more cases of fever and/or respiratory symptoms in a small area (family, office, school class, etc.) within 2 week 
Figure 1. Quarantine decision algorithm.

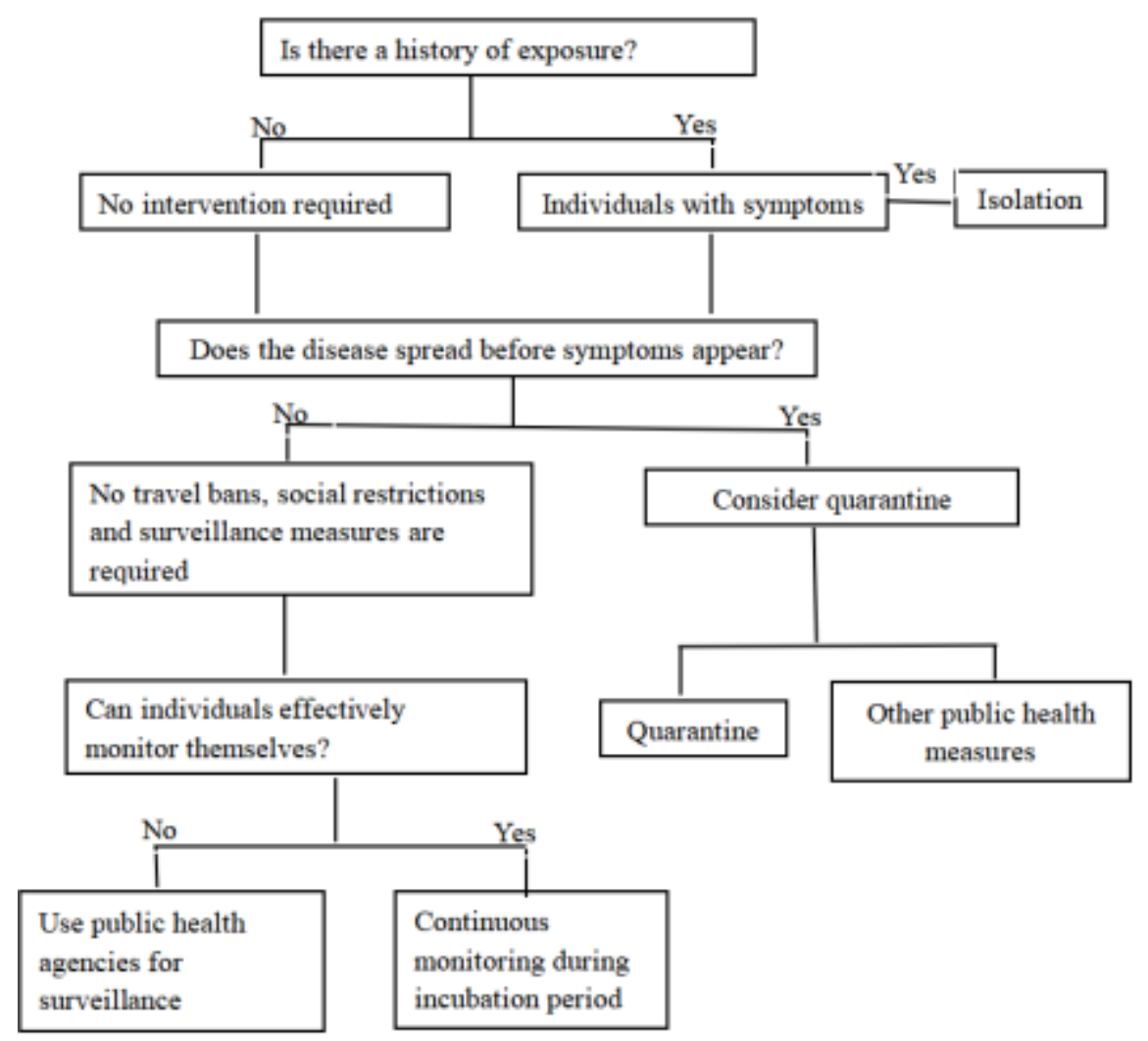

